\title{
Inventory System with Shortages and Weibull Deterioration Purpose
}

\author{
Kapil Kumar Bansal, Ompal Singh, Satish Kumar, Priti Chaudhary
}

\begin{abstract}
In the paper, a venture has been made to develop a stock presentation for interminable planning horizon with exponentially growing interest value. It might be seen that debilitating doesn't depend on time as it were. It can impact as a result of climate conditions, clamminess, and capacity conditions, etc in this way it is progressively reasonable to consider rot rate as two-parameter Weibull spread work. Inadequacy is allowed and totally multiplied. The holding cost contemplated a direct limit of time. The ideal solution of the proposed stock show is construed and pondered same cases.
\end{abstract}

Keywords: Inventory System, Deterioration, Weibull distribution.

\section{INTRODUCTION}

The two-warehouse model can be connected to numerous down to earth circumstances, because of the presence of open market strategy; the business rivalry turns out to be extremely high to involve the most extreme conceivable market. Subsequently, the administration of the departmental store is limited to contract a different warehouse on rental premise at a separation place for putting away of abundance things. Complete accumulated deficiencies are allowed in this investigation.

The models are quite useful in practice. In the previously, many authors have considered inventory models for diminishing items stored in two warehouses. Sarma (1987) A deterministic inventory model for deteriorating items with two storage facilities. Pakkala and Achary (1992a) considered discrete time inventory model for deteriorating items with two warehouses. Yang (2006) studied model with inflation in which shortages are considered to be partially backlogged. Dye et al. (2007) have dealt with time proportional backlogging. Everyone of these papers accept a constant interest rate. Circumstances, there are items like unpredictable fluids, drugs, and materials, and so on in which the rate of weakening is expansive. Hence, the misfortune because of disintegration ought not to be disregarded. Aggarwal and Jaggi (1995) broadened Goyal's (2015) model to consider breaking down things. Deficiencies are of incredible significance particularly in a model that considers a postponement in installment because of the way that deficiencies can influence the quantity ordered to profit by the deferral in the chapter.

Revised Manuscript Received on March 17, 2020.

* Correspondence Author

Dr.Kapil Kumar Bansal*, Department of Mathematics, SRM Institute of Science \& Technology, Ghaziabad-201204, India. E-mail: drkapilbansal25@gmail.com

Dr.Ompal Singh, Department of Mathematics, SRM Institute of Science \& Technology, Ghaziabad-201204, India

Dr.Satish Kumar, Department of Mathematics, SRM Institute of Science \& Technology,Ghaziabad-201204, India

Priti Chaudhary, Department of Mathematics, SRM Institute of Science \& Technology, Ghaziabad-201204, India

(C) The Authors. Published by Blue Eyes Intelligence Engineering and Sciences Publication (BEIESP). This is an open access article under the CC BY-NC-ND license (http://creativecommons.org/licenses/by-nc-nd/4.0/)
Singh et al. (2008) introduced there are 2-weibull distribution inventory demonstrate for decaying things with consistent interest rate where deficiencies were permitted and somewhat multiplied. Singh and Jain (2009) proposed a deterministic inventory show with time shifting disintegration rate and a straight pattern sought after over a finite planning skyline. They accepted that the provider offers a credit cutoff to the retailer amid which no intrigue is charged.

Covert and Philip (1973) 'An EOQ model for items with Weibull distribution deterioration'. Ghare and Schrader's (1963) display and get a financial order quantity show for a variable rate of crumbling by expecting a two-parameter Weibull appropriation, Philip (2014) created EOQ models for things with variable rate of weakening which was additionally summed up by Shah (2015) permitting deficiencies and considering general falling apart capacities. The impact of expansion on inventory management has analyzed by a few creators. Moreover, as discussed in Chakrabarty et al. (1998), An EOQ model for items with Weibull distribution deterioration, shortages and trended demand.

In the paper, we have attempted to build up a twowarehouse inventory framework with a sensible and down to earth disintegration rate. The impact of crumbling of physical merchandise in stock is a sensible component's inventory control. In this model crumbling rate at anything is expected to pursue two-parameter Weibull dispersion capacity of time. This decay rate is appropriate for things with and without life-period. The two warehouse inventory problem is a captivating yet viable subject of choice science.

1.1 Assumptions and Notation
i)
The reviving size is consistent and creation is
prompt in the midst of supported time span $\mathrm{T}$ of
each cycle.
Lead time is 0 .
ii)
iii)
aggregated.
iv) Demand rate $\mathrm{D}(\mathrm{t})=\frac{\mathrm{d}}{(\mathrm{e}-1) \mathrm{T}} \mathrm{e}^{\frac{\mathrm{t}}{\mathrm{T}}}$ at a time $\mathrm{t}$.
v) $\quad \begin{aligned} & \text { Deterioration rate } \theta=\alpha \beta \mathrm{t}^{\beta-1} \\ & \text {, where } 0<\alpha<1\end{aligned}$
vi) $\mathrm{C}_{1}, \mathrm{C}_{2}$ are cost of each item, shortage cost per unit
time respectively.
vii)
1.2 Mathematical Modeling and Analysis for the System

Let $\mathrm{I}(\mathrm{t})$ is the current stock stage at a time $\mathrm{t}$.

$$
\frac{\mathrm{dI}(\mathrm{t})}{\mathrm{dt}}+\theta \mathrm{I}(\mathrm{t})=-\frac{\mathrm{d}}{(\mathrm{e}-1) \mathrm{T}} \mathrm{e}^{\frac{\mathrm{t}}{\mathrm{T}}}, \quad 0 \leq \mathrm{t} \leq \mathrm{t}_{1}
$$




$$
\begin{array}{r}
\frac{d I(t)}{d t}=-\frac{d}{(e-1) T} e^{\frac{t}{T},} \quad t_{1} \leq t \\
\leq T
\end{array}
$$

Equation (1.1)

$$
\begin{gathered}
\frac{d I(t)}{d t}+\alpha \beta t^{\beta-1} \cdot I(t)=-\frac{d}{(e-1)} e^{\frac{t}{T}} \\
\text { I.F. }=e^{\int \alpha \beta t^{\beta-1} d t}=e^{\alpha t^{\beta}} .
\end{gathered}
$$

Solution of equation (1.1) is given by

$$
I(t) e^{\alpha t^{\beta}}=-\int \frac{d}{(e-1) T} e^{\frac{t}{T}} e^{\alpha t^{\beta}} d t+B
$$

Where "B" the of integration

$$
=-\frac{d}{(e-1) T} \int e^{\frac{t}{T}}\left(1+\alpha t^{\beta}\right) d t+B \text {. }
$$

After expanding $\mathrm{e}^{\frac{\mathrm{t}}{\mathrm{T}}}$ by Taylor's series and neglecting higher order terms of

$$
\begin{aligned}
& \frac{\mathrm{t}}{\mathrm{T}} \text { greater than } 1\left(\because \frac{\mathrm{t}}{\mathrm{T}}<1\right)=\text { we get } \\
& \quad \begin{array}{r}
\mathrm{I}(\mathrm{t}) \mathrm{e}^{\alpha \mathrm{t}^{\beta}}=-\frac{\mathrm{d}}{(\mathrm{e}-1) \mathrm{T}} \int\left(1+\frac{\mathrm{t}}{\mathrm{T}}\right)\left(1+\alpha \mathrm{t}^{\beta}\right) \mathrm{dt}+\mathrm{B} \\
=-\frac{\mathrm{d}}{(\mathrm{e}-1) \mathrm{T}} \int\left(1+\frac{\mathrm{t}}{\mathrm{T}}+\alpha \mathrm{t}^{\beta}+\frac{\alpha}{\mathrm{T}} \mathrm{t}^{\beta+1}\right) \mathrm{dt}+\mathrm{B} \\
=-\frac{\mathrm{d}}{(\mathrm{e}-1) \mathrm{T}}\left[\mathrm{t}+\frac{\mathrm{t}^{2}}{2 \mathrm{~T}}+\frac{\alpha \mathrm{t}^{\beta+1}}{\beta+1}+\frac{\alpha \mathrm{t}^{\beta+2}}{\mathrm{~T}(\beta+1)}\right] \\
+\mathrm{B} . \quad \ldots \ldots \ldots(1.3)
\end{array}
\end{aligned}
$$

At $\mathbf{t}=\mathbf{O}, \quad \mathbf{I}(\mathbf{t})=\mathbf{S}$, then from equation (1.3), we have

$$
I(t) e^{\alpha t^{\beta}}=-\frac{d}{(e-1) T}\left[t+\frac{t^{2}}{2 T}+\frac{\alpha t^{\beta+1}}{\beta+1}+\frac{\alpha t^{\beta+2}}{T(\beta+2)}\right]+S .
$$

At $t=t_{1}, I(t)=0$, then from equation (84), we have

$$
S=\frac{d}{(e-1) T}\left[t_{1}+\frac{t_{1}^{2}}{2 T}+\frac{\alpha t_{1}^{\beta+1}}{\beta+1}+\frac{\alpha t_{1}^{\beta+2}}{T(\beta+2)}\right] .
$$

Substituting the value of $S$ in equation (1.4) from equation (1.5), then

$$
\begin{aligned}
I(t)=\frac{d}{(e-1) T}\left[t_{1}\right. & +\frac{t_{1}^{2}}{2 T}+\frac{\alpha t_{1}^{\beta+1}}{\beta+1}+\frac{\alpha t_{1}^{\beta+2}}{T(\beta+2)}-t \\
& \left.-\frac{t^{2}}{2 T}-\frac{\alpha t^{\beta+1}}{\beta+1}-\frac{\alpha t^{\beta+2}}{T(\beta+2)}\right]\left(1-\alpha t^{\beta}\right)
\end{aligned}
$$

Neglecting higher order terms of $\alpha$, we get from equation (1.6)

$$
\begin{aligned}
& I(t)=\frac{d}{(e-1) T}\left[t_{1}-t+\frac{t_{1}^{2}}{2 T}-\frac{t^{2}}{2 T}-\alpha t_{1} t^{\beta}+\alpha t^{\beta+1}-\frac{\alpha t_{1}^{2} t^{\beta}}{2 T}\right. \\
& \left.+\frac{\alpha t^{\beta+2}}{2 T}+\frac{\alpha t_{1}^{\beta+1}}{\beta+1}+\frac{\alpha t_{1}^{\beta+2}}{T(\beta+2)}-\frac{\alpha t^{\beta+1}}{\beta+1}-\frac{\alpha t^{\beta+2}}{T(\beta+2)}\right]
\end{aligned}
$$

$$
\begin{aligned}
& I(t)=\frac{d}{(\ell-1) T}\left[t_{1}+\frac{t_{1}^{2}}{2 T}+\frac{\alpha t_{1}^{\beta+1}}{\beta+1}+\frac{\alpha t_{1}^{\beta+2}}{T(\beta+2)}-t\right. \\
& \left.-\frac{t^{2}}{2 T}-\alpha t_{1} t^{\beta}-\frac{\alpha t_{1}^{2} t^{\beta}}{2 T}+\frac{\alpha \beta t^{\beta+1}}{\beta+1}+\frac{\alpha \beta t^{\beta+2}}{2 T(\beta+2)}\right]
\end{aligned}
$$

Solution of equation (1.2) is given by

$$
I(t)=\frac{d}{e-1}\left(e^{\frac{t_{1}}{T}}-e^{\frac{t}{T}}\right) .
$$

Total amount of deteriorated units

$$
D=S-\int_{0}^{t_{1}} \frac{d}{(e-1) T} e^{\frac{t}{T}} d t
$$

$$
\begin{aligned}
=S- & \frac{d}{(e-1) T}\left[T e^{\frac{t}{T}}\right]_{0}^{t_{1}} \\
= & S-\frac{d}{(e-1)}\left(e^{\frac{t_{1}}{T}}-1\right)
\end{aligned}
$$

Putting the value of $S$ in equation (1.6), we obtain

$$
\mathrm{D}=\frac{\mathrm{d}}{(\mathrm{e}-1) \mathrm{T}}\left[\mathrm{t}_{1}+\frac{\mathrm{t}_{1}^{2}}{2 \mathrm{~T}}+\frac{\alpha \mathrm{t}_{1}^{\beta+1}}{\beta+1}+\frac{\alpha \mathrm{t}_{1}^{\beta+2}}{\mathrm{~T}(\beta+2)}-\mathrm{Te}^{\frac{\mathrm{t}_{1}}{\mathrm{~T}}}+\mathrm{T}\right]
$$

Number of units in shortage

$$
\begin{aligned}
& =\int_{\mathrm{t}_{1}}^{\mathrm{T}} \mathrm{I}(\mathrm{t}) \mathrm{dt} \\
& =\frac{\mathrm{d}}{\mathrm{e}-1}\left[2 \mathrm{Te}^{\frac{\mathrm{t}_{1}}{\mathrm{~T}}}-\mathrm{t}_{1} \mathrm{e}^{\frac{\mathrm{t}_{1}}{\mathrm{~T}}}-\mathrm{eT}\right] .
\end{aligned}
$$

Therefore average shortage cost

$$
=\frac{\mathrm{C}_{2} \mathrm{~d}}{\mathrm{~T}(\mathrm{e}-1)}\left[2 \mathrm{Te}^{\frac{\mathrm{t}_{1}}{\mathrm{~T}}}-\mathrm{t}_{1} \mathrm{e}^{\frac{\mathrm{t}_{1}}{\mathrm{~T}}}-\mathrm{eT}\right]
$$

Average holding cost

$$
\begin{aligned}
& =\frac{1}{T} \int_{0}^{t_{1}} I(t)(h+\gamma t) \cdot d t \\
& =\frac{1}{T} \int_{0}^{t_{1}} h I(t) d t+\frac{1}{T} \int_{0}^{t_{1}} \gamma t I(t) d t
\end{aligned}
$$

Published By:

Blue Eyes Intelligence Engineering

\& Sciences Publication

(C) Copyright: All rights reserved. 


$$
\begin{aligned}
& =\frac{\mathrm{h}}{\mathrm{T}} \frac{\mathrm{d}}{(\mathrm{e}-1) \mathrm{T}} \int_{0}^{\mathrm{t}_{1}}\left[\mathrm{t}_{1}+\frac{\mathrm{t}_{1}^{2}}{2 \mathrm{~T}}+\frac{\alpha \mathrm{t}_{1}^{\beta+1}}{\beta+1}+\frac{\alpha \mathrm{t}_{1}^{\beta+2}}{\mathrm{~T}(\beta+2)}-\mathrm{t}-\frac{\mathrm{t}^{2}}{2 \mathrm{~T}}\right. \\
& \left.-\alpha t_{1} t^{\beta}-\frac{\alpha t_{1}^{2} t^{\beta}}{2 T}+\frac{\alpha \beta t^{\beta+1}}{\beta+1}+\frac{\alpha \beta t^{\beta+2}}{2 T(\beta+2)}\right] d t \\
& +\frac{\gamma}{T} \frac{d}{(e-1) T} \int_{0}^{t_{1}}\left[t_{1} t+\frac{t_{1}^{2} t}{2 T}+\frac{\alpha t_{1}^{\beta+1} \cdot t}{\beta+1}+\frac{\alpha t_{1}^{\beta+2}}{T(\beta+2)} t-t^{2}\right. \\
& \left.-\frac{t^{3}}{2 T}-\alpha t_{1} t^{\beta+1}-\frac{\alpha t_{1}^{2} t^{\beta+1}}{2 T}+\frac{\alpha \beta t^{\beta+2}}{\beta+1}+\frac{\alpha \beta t^{\beta+3}}{2 T(\beta+2)}\right] d t \\
& =\frac{h}{T} \frac{d}{(e-1) T}\left\lceil t_{1}^{2}+\frac{t_{1}^{3}}{2 T}+\frac{\alpha t_{1}^{\beta+2}}{\beta+1}+\frac{\alpha t_{1}^{\beta+3}}{T(\beta+2)}-\frac{t_{1}^{2}}{2}-\frac{t_{1}^{3}}{6 T}\right. \\
& \left.-\frac{\alpha \mathrm{t}_{1}^{\beta+2}}{\beta+2}-\frac{\alpha \mathrm{t}_{1}^{\beta+3}}{2 \mathrm{~T}(\beta+1)}+\frac{\alpha \beta \mathrm{t}_{1}^{\beta+2}}{(\beta+1)(\beta+2)}+\frac{\alpha \beta_{1}^{\beta+3}}{2 \mathrm{~T}(\beta+2)(\beta+3)}\right] \\
& +\frac{\gamma \mathrm{d}}{\mathrm{T}(\mathrm{e}-1) \mathrm{T}}\left[\frac{\mathrm{t}_{1}^{3}}{2}+\frac{\mathrm{t}_{1}^{4}}{4 \mathrm{~T}}+\frac{\alpha \mathrm{t}_{1}^{\beta+3}}{2(\beta+1)}+\frac{\alpha \mathrm{t}_{1}^{\beta+4}}{2 \mathrm{~T}(\beta+2)}-\frac{\mathrm{t}_{1}^{3}}{3}\right. \\
& \left.-\frac{t_{1}^{4}}{8 \mathrm{~T}}-\frac{\alpha \mathrm{t}_{1}^{\beta+3}}{\beta+2}-\frac{\alpha \mathrm{t}_{1}^{\beta+4}}{2 \mathrm{~T}(\beta+2)}+\frac{\alpha \beta \mathrm{t}_{1}^{\beta+3}}{(\beta+1)(\beta+3)}+\frac{\alpha \beta \mathrm{t}_{1}^{\beta+4}}{2 \mathrm{~T}(\beta+2)(\beta+4)}\right] \\
& =\frac{\mathrm{h}}{\mathrm{T}^{2}} \frac{\mathrm{d}}{\mathrm{e}-1}\left[\frac{\mathrm{t}_{1}^{2}}{2}+\frac{\mathrm{t}_{1}^{3}}{3 \mathrm{~T}}+\frac{\alpha \beta \mathrm{t}_{1}^{\beta+3}}{2 \mathrm{~T}(\beta+1)(\beta+2)}+\frac{\alpha \beta \mathrm{t}_{1}^{\beta+2}}{(\beta+1)(\beta+2)}\right. \\
& \left.+\frac{\alpha \beta \mathrm{t}_{1}^{\beta+3}}{2 \mathrm{~T}(\beta+2)(\beta+3)}\right]+\frac{\mathrm{y}}{\mathrm{T}^{2}} \frac{\mathrm{d}}{\mathrm{e}-1}\left[\frac{\mathrm{t}_{1}^{3}}{6}+\frac{\mathrm{t}_{1}^{4}}{8 \mathrm{~T}}-\frac{\alpha \beta \mathrm{t}_{1}^{\beta+3}}{2(\beta+1)(\beta+2)}\right. \\
& \left.+\frac{\alpha \beta t_{1}^{\beta+3}}{(\beta+1)(\beta+3)}+\frac{\alpha \beta t_{1}^{\beta+4}}{2 \mathrm{~T}(\beta+2)(\beta+4)}\right] \\
& =\frac{\mathrm{hd}}{\mathrm{T}^{2}(\mathrm{e}-1)}\left[\frac{\mathrm{t}_{1}^{2}}{2}+\frac{\mathrm{t}_{1}^{3}}{3 \mathrm{~T}}+\frac{\alpha \beta \mathrm{t}_{1}^{\beta+3}}{\mathrm{~T}(\beta+1)(\beta+3)}+\frac{\alpha \beta \mathrm{t}_{1}^{\beta+2}}{(\beta+1)(\beta+2)}\right. \\
& +\frac{\gamma d}{T^{2}(e-1)}\left[\frac{t_{1}^{3}}{6}+\frac{t_{1}^{4}}{8 T}+\frac{\alpha \beta t_{1}^{\beta+3}}{2(\beta+2)(\beta+3)}+\frac{\alpha \beta t_{1}^{\beta+4}}{2 T(\beta+2)(\beta+4)}\right] .
\end{aligned}
$$

Total average cost per time is

$$
\mathrm{K}\left(\mathrm{T}_{1}\right)=\frac{\mathrm{CD}}{\mathrm{T}}+{ }_{\text {Average Holding cost }+ \text { Average }}
$$

Shortage cost

$$
\begin{aligned}
& =\frac{\mathrm{CD}}{\mathrm{T}}+\frac{\mathrm{hd}}{\mathrm{T}^{2}(\mathrm{e}-1)}\left[\frac{\mathrm{t}_{1}^{2}}{2}+\frac{\mathrm{t}_{1}^{3}}{3 \mathrm{~T}}+\frac{\alpha \beta \mathrm{t}_{1}^{\beta+3}}{\mathrm{~T}(\beta+1)(\beta+3)}+\frac{\alpha \beta \mathrm{t}_{1}^{\beta+2}}{(\beta+1)(\beta+2)}\right] \\
& +\frac{\gamma \mathrm{d}}{\mathrm{T}^{2}(\mathrm{e}-1)}\left[\frac{\mathrm{t}_{1}^{3}}{6}+\frac{\mathrm{t}_{1}^{4}}{8 \mathrm{~T}}+\frac{\alpha \beta \mathrm{t}_{1}^{\beta+3}}{2(\beta+1)(\beta+3)}+\frac{\alpha \beta \mathrm{t}_{1}^{\beta+4}}{2 \mathrm{~T}(\beta+1)(\beta+2)}\right] \\
& +\frac{\mathrm{C}_{2} \mathrm{~d}}{\mathrm{~T}(\mathrm{e}-1)}\left(2 \mathrm{Te}^{\frac{\mathrm{t}_{1}}{\mathrm{~T}}}-\mathrm{t}_{1} \mathrm{e}^{\frac{\mathrm{t}_{1}}{\mathrm{~T}}}-\mathrm{eT}\right) \\
& \mathrm{K}\left(\mathrm{T}_{1}\right)=\frac{\mathrm{cd}}{\mathrm{T}^{2}(\mathrm{e}-1)}\left[\mathrm{t}_{1}+\frac{\mathrm{t}_{1}^{2}}{2 \mathrm{~T}}+\frac{\alpha \mathrm{t}_{1}^{\beta+1}}{\beta+1}+\frac{\alpha \mathrm{t}_{1}^{\beta+2}}{\mathrm{~T}(\beta+2)}-\mathrm{Te}^{\frac{\mathrm{t}_{1}}{\mathrm{~T}}}+\mathrm{T}\right]
\end{aligned}
$$

$$
\begin{aligned}
& +\frac{\mathrm{hd}}{\mathrm{T}^{2}(\mathrm{e}-1)}\left[\frac{\mathrm{t}_{1}^{2}}{2}+\frac{\mathrm{t}_{1}^{3}}{3 \mathrm{~T}}+\frac{\alpha \beta \mathrm{t}_{1}^{\beta+3}}{\mathrm{~T}(\beta+1)(\beta+3)}+\frac{\alpha \beta \mathrm{t}_{1}^{\beta+2}}{(\beta+1)(\beta+2)}\right] \\
& +\frac{\gamma \mathrm{d}}{\mathrm{T}^{2}(\mathrm{e}-1)}\left[\frac{\mathrm{t}_{1}^{3}}{6}+\frac{\mathrm{t}_{1}^{4}}{8 \mathrm{~T}}+\frac{\alpha \beta \mathrm{t}_{1}^{\beta+3}}{2(\beta+2)(\beta+3)}+\frac{\alpha \beta \mathrm{t}_{1}^{\beta+4}}{2 \mathrm{~T}(\beta+2)(\beta+4)}\right] \\
& +\frac{\mathrm{c}_{2} \mathrm{~d}}{\mathrm{~T}(\mathrm{e}-1)}\left(2 \mathrm{Te}^{\frac{\mathrm{t}_{1}}{\mathrm{~T}}}-\mathrm{t}_{1} \mathrm{e}^{\frac{\mathrm{t}_{1}}{\mathrm{~T}}}-\mathrm{eT}\right)
\end{aligned}
$$

The necessary conditions for minimum the total average costs $\mathrm{K}\left(\mathrm{t}_{1}, \mathrm{~T}\right)$ are

$$
\frac{\partial \mathrm{K}}{\partial \mathrm{T}}=0 \quad \text { And } \quad \frac{\partial \mathrm{K}}{\partial \mathrm{t}_{1}}=0
$$

Now $\frac{\partial \mathrm{K}}{\partial \mathrm{T}}=0$, gives

$$
\begin{aligned}
& -2 C\left[t_{1}+\frac{3 t_{1}^{2}}{4 T}+\frac{\alpha t_{1}^{\beta+1}}{\beta+1}+\frac{3 \alpha t_{1}^{\beta+2}}{2 T(\beta+2)}-\frac{T^{\frac{t_{1}}{T}}}{2}-\frac{t_{1} e^{\frac{t_{1}}{T}}}{2}+\frac{T}{2}\right] \\
& -3 h\left[\frac{t_{1}^{2}}{3}+\frac{t_{1}^{3}}{3 T}+\frac{\alpha \beta t_{1}^{\beta+3}}{T(\beta+1)(\beta+3)}+\frac{2 \alpha \beta t_{1}^{\beta+2}}{3(\beta+1)(\beta+2)}\right] \\
& -3 \gamma\left[\frac{2 t_{1}^{3}}{9}+\frac{t_{1}^{4}}{8 T}+\frac{\alpha \beta t_{1}^{\beta+3}}{2(\beta+2)(\beta+3)}+\frac{\alpha \beta t_{1}^{\beta+4}}{2 T(\beta+2)(\beta+4)}\right] \\
& +C_{2} t_{1}^{2} e^{\frac{t_{1}}{T}}=0 .
\end{aligned}
$$$$
\text { .......(1.15) }
$$

And $\frac{\partial \mathrm{K}}{\partial \mathrm{t}_{1}}=0$, gives

$C\left[1+\frac{t_{1}}{T}+\alpha t_{1}^{\beta}+\frac{\alpha t_{1}^{\beta+1}}{T}-e^{\frac{t_{1}}{T}}\right]$

$+h\left[t_{1}+\frac{t_{1}^{2}}{T}+\frac{\alpha \beta t_{1}^{\beta+1}}{\beta+1}+\frac{\alpha \beta t_{1}^{\beta+2}}{T(\beta+1)}\right]$

$+\gamma\left[\frac{\mathbf{t}_{1}^{2}}{2}+\frac{\mathbf{t}_{1}^{3}}{2 \mathrm{~T}}+\frac{\alpha \beta \mathbf{t}_{1}^{\beta+2}}{2(\beta+2)}+\frac{\alpha \beta \mathbf{t}_{1}^{\beta+3}}{2 \mathrm{~T}(\beta+2)}\right]$

$+C_{2}\left(T e^{\frac{t_{1}}{T}}-\frac{t_{1}}{T} e^{\frac{t_{1}}{T}}\right)=0$.

Equation (1.15) and (1.16) gives the optimum values of $\mathrm{T}$ and $t_{1}$ respectively. Provided

$\frac{\partial^{2} \mathrm{~K}}{\partial \mathrm{T}^{2}}>0, \quad \frac{\partial^{2} \mathrm{~K}}{\partial \mathrm{t}_{1}^{2}}>0 \quad$ and $\left(\frac{\partial^{2} \mathrm{~K}}{\partial \mathrm{T}^{2}}\right)\left(\frac{\partial^{2} \mathrm{~K}}{\partial \mathrm{t}_{1}^{2}}\right)-\left(\frac{\partial^{2} \mathrm{~K}}{\partial \mathrm{T} \partial \mathrm{t}_{1}}\right)^{2}>0$

...(1.17)

Case I. in case of finite planning horizon, the complete average cost is

$$
\begin{aligned}
& \mathrm{K}\left(\mathrm{t}_{1}\right)=\frac{\mathrm{Cd}}{\mathrm{T}^{2}(\mathrm{e}-1)}\left[\mathrm{t}_{1}+\frac{\mathrm{t}_{1}^{2}}{2 \mathrm{~T}}+\frac{\alpha \mathrm{t}_{1}^{\beta+1}}{\beta+1}+\frac{\alpha \mathrm{t}_{1}^{\beta+2}}{\mathrm{~T}(\beta+2)}-\mathrm{Te}^{\frac{\mathrm{t}_{1}}{\mathrm{~T}}}+\mathrm{T}\right] \\
& +\frac{\mathrm{hd}}{\mathrm{T}^{2}(\mathrm{e}-1)}\left[\frac{\mathrm{t}_{1}^{2}}{2}+\frac{\mathrm{t}_{1}^{3}}{3 \mathrm{~T}}+\frac{\alpha \beta \mathrm{t}_{1}^{\beta+3}}{\mathrm{~T}(\beta+1)(\beta+3)}+\frac{\alpha \beta \mathrm{t}_{1}^{\beta+2}}{\mathrm{~T}(\beta+1)(\beta+2)}\right] \\
& +\frac{\gamma \mathrm{d}}{\mathrm{T}^{2}(\mathrm{e}-1)}\left[\frac{\mathrm{t}_{1}^{3}}{6}+\frac{\mathrm{t}_{1}^{4}}{8 \mathrm{~T}}+\frac{\alpha \beta \mathrm{t}_{1}^{\beta+3}}{2(\beta+2)(\beta+3)}+\frac{\alpha \beta \mathrm{t}_{1}^{\beta+4}}{2 \mathrm{~T}(\beta+2)(\beta+4)}\right] \\
& +\frac{\mathrm{C}_{2} \mathrm{~d}}{\mathrm{~T}(\mathrm{e}-1)}\left[2 \mathrm{Te}^{\frac{\mathrm{t}_{1}}{\mathrm{~T}}}-\mathrm{t}_{1} \mathrm{e}^{\frac{\mathrm{t}_{1}}{\mathrm{~T}}}-\mathrm{e}\right]
\end{aligned}
$$

Published By:

Blue Eyes Intelligence Engineering

\& Sciences Publication

(C) Copyriaht: All rights reserved. 
Sub- case 1. When $\beta=1$ then average cost is

$\mathrm{K}\left(\mathrm{t}_{1}\right)=\frac{c d}{\mathrm{~T}^{2}(\mathrm{e}-1)}\left[\mathrm{t}_{1}+\frac{\mathrm{t}_{1}^{2}}{2 \mathrm{~T}}+\frac{\alpha \mathrm{t}_{1}^{2}}{2}+\frac{\alpha \mathrm{t}_{1}^{3}}{3 \mathrm{~T}}-\mathrm{Te}^{\frac{\mathrm{t}_{1}}{\mathrm{~T}}}+\mathrm{T}\right]$

$+\frac{\mathrm{hd}}{\mathrm{T}^{2}(\mathrm{e}-1)}\left[\frac{\mathrm{t}_{1}^{2}}{2}+\frac{\mathrm{t}_{1}^{3}}{3 \mathrm{~T}}+\frac{\alpha \mathrm{t}_{1}^{4}}{8 \mathrm{~T}}+\frac{\alpha \mathrm{t}_{1}^{3}}{6}\right]+\frac{\gamma \mathrm{d}}{\mathrm{T}^{2}(\mathrm{e}-1)}$

$\left[\frac{\mathrm{t}_{1}^{3}}{6}+\frac{\mathrm{t}_{1}^{4}}{8 \mathrm{~T}}+\frac{\alpha \mathrm{t}_{1}^{4}}{24}+\frac{\alpha \mathrm{t}_{1}^{5}}{30 \mathrm{~T}}\right]+\frac{\mathrm{c}_{2} \mathrm{~d}}{\mathrm{~T}(\mathrm{e}-1)}\left(2 \mathrm{Te}^{\frac{\mathrm{t}_{1}}{\mathrm{~T}}}-\mathrm{t}_{1} \mathrm{e}^{\frac{\mathrm{t}_{1}}{\mathrm{~T}}}-\mathrm{eT}\right)$

$\frac{\mathrm{dK}\left(\mathrm{t}_{1}\right)}{\mathrm{dt}_{1}}=\frac{\mathrm{cd}}{\mathrm{T}^{2}(\mathrm{e}-1)}\left[1+\frac{\mathrm{t}_{1}}{\mathrm{~T}}+\alpha \mathrm{t}_{1}+\frac{\alpha \mathrm{t}_{1}^{2}}{\mathrm{~T}}-\mathrm{e}^{\frac{\mathrm{t}_{1}}{\mathrm{~T}}}\right]$

$+\frac{h d}{T^{2}(e-1)}\left[t_{1}+\frac{t_{1}^{2}}{T}+\frac{\alpha t_{1}^{3}}{2 T}+\frac{\alpha t_{1}^{2}}{2}\right]+\frac{\gamma d}{T^{2}(e-1)}$

$\left[\frac{t_{1}^{2}}{2}+\frac{t_{1}^{3}}{2 T}+\frac{\alpha t_{1}^{3}}{6}+\frac{\alpha t_{1}^{4}}{6 T}\right]+\frac{c_{2} d}{T^{2}(e-1)}\left(\mathrm{Te}^{\frac{t_{1}}{T}}-t_{1} e^{\frac{t_{1}}{T}}\right)$

For minimum total average cost $\frac{\mathrm{dK}\left(\mathrm{t}_{1}\right)}{\mathrm{dt}_{1}}=\mathbf{0}$

$\Rightarrow \quad C\left(1+\frac{t_{1}}{T}+\alpha t_{1}+\frac{\alpha t_{1}^{2}}{T}-e^{\frac{t_{1}}{T}}\right)+h\left(t_{1}+\frac{t_{1}^{2}}{T}+\frac{\alpha t_{1}^{3}}{2 T}+\frac{\alpha t_{1}^{2}}{2}\right)$

$+\gamma\left(\frac{t_{1}^{2}}{2}+\frac{t_{1}^{3}}{2 T}+\frac{\alpha t_{1}^{3}}{6}+\frac{\alpha t_{1}^{4}}{6 T}\right)+c_{2}\left(T e^{\frac{t_{1}}{T}}-t_{1} e^{\frac{t_{1}}{T}}\right)=0$

Sub- case 2 . When $\beta=2$, deteriration rate become variable linear function of time then total average cost is

$$
\begin{aligned}
& \mathrm{K}\left(\mathrm{t}_{1}\right)=\frac{\mathrm{Cd}}{\mathrm{T}^{2}(\mathrm{e}-1)}\left[\mathrm{t}_{1}+\frac{\mathrm{t}_{1}^{2}}{2 \mathrm{~T}}+\frac{\alpha \mathrm{t}_{1}^{3}}{3}+\frac{\alpha \mathrm{t}_{1}^{4}}{4 \mathrm{~T}}-\mathrm{Te}^{\frac{\mathrm{t}_{1}}{\mathrm{~T}}}+\mathrm{T}\right] \\
& +\frac{\mathrm{hd}}{\mathrm{T}^{2}(\mathrm{e}-1)}\left[\frac{\mathrm{t}_{1}^{2}}{2}+\frac{\mathrm{t}_{1}^{3}}{3 \mathrm{~T}}+\frac{2 \alpha \mathrm{t}_{1}^{5}}{15 \mathrm{~T}}+\frac{\alpha \mathrm{t}_{1}^{4}}{6}\right]+\frac{\gamma \mathrm{d}}{\mathrm{T}^{2}(\mathrm{e}-1)} \\
& {\left[\frac{\mathrm{t}_{1}^{3}}{6}+\frac{\mathrm{t}_{1}^{4}}{8 \mathrm{~T}}+\frac{\alpha \mathrm{t}_{1}^{5}}{20}+\frac{\alpha \mathrm{t}_{1}^{6}}{24 \mathrm{~T}}\right]+\frac{\mathrm{c}_{2} \mathrm{~d}}{\mathrm{~T}^{2}(\mathrm{e}-1)}\left(2 \mathrm{~T}^{2} \mathrm{e}^{\frac{\mathrm{t}_{1}}{\mathrm{~T}}}-\mathrm{t}_{1} \mathrm{Te}^{\frac{\mathrm{t}_{1}}{\mathrm{~T}}}-\mathrm{eT}^{2}\right)}
\end{aligned}
$$

For minimum value of $\mathrm{K}\left(\mathrm{t}_{1}\right)$, $\frac{\mathrm{dK}\left(\mathrm{t}_{1}\right)}{\mathrm{dt}_{1}}=\mathrm{O}$

$\Rightarrow \quad C\left(1+\frac{t_{1}}{T}+\alpha t_{1}^{2}+\frac{\alpha t_{1}^{3}}{T}-e^{\frac{t_{1}}{T}}\right)+h\left(t_{1}+\frac{t_{1}^{2}}{T}+\frac{2 \alpha t_{1}^{4}}{3 T}+\frac{2 \alpha t_{1}^{3}}{3}\right)$

$+\gamma\left(\frac{t_{1}^{2}}{2}+\frac{t_{1}^{3}}{2 \mathrm{~T}}+\frac{\alpha t_{1}^{4}}{4}+\frac{\alpha t_{1}^{5}}{4 \mathrm{~T}}\right)+\mathrm{c}_{2}\left(\mathrm{Te}^{\frac{\mathrm{t}_{1}}{\mathrm{~T}}}-\mathrm{t}_{1} \mathrm{e}^{\frac{\mathrm{t}_{1}}{\mathrm{~T}}}\right)=0$

Sub-case 3 . When $\beta=3$, then deterioration rate become quadratic function of time then toatl average cost is

$\mathrm{K}\left(\mathrm{t}_{1}\right)=\frac{\mathrm{Cd}}{\mathrm{T}^{2}(\mathrm{e}-1)}\left[\mathrm{t}_{1}+\frac{\mathrm{t}_{1}^{2}}{2 \mathrm{~T}}+\frac{\alpha \mathrm{t}_{1}^{4}}{4}+\frac{\alpha \mathrm{t}_{1}^{5}}{5 \mathrm{~T}}-\mathrm{Te}^{\frac{\mathrm{t}_{1}}{\mathrm{~T}}}+\mathrm{T}\right]$

$+\frac{h d}{T^{2}(e-1)}\left[\frac{t_{1}^{2}}{2}+\frac{t_{1}^{3}}{3 T}+\frac{\alpha t_{1}^{6}}{8 T}+\frac{3 \alpha t_{1}^{5}}{20}\right]+\frac{\gamma d}{T^{2}(e-1)}$

$\left[\frac{t_{1}^{3}}{6}+\frac{t_{1}^{4}}{8 T}+\frac{\alpha t_{1}^{6}}{2 O}+\frac{3 \alpha t_{1}^{7}}{7 O T}\right]+\frac{c_{2} d}{T(e-1)}\left[2 \mathrm{Te}^{\frac{t_{1}}{T}}-t_{1} e^{\frac{t_{1}}{T}}-e T\right]$

For minimum value of $\mathrm{K}\left(\mathrm{t}_{1}\right)$, $\frac{\mathrm{dK}\left(\mathrm{t}_{1}\right)}{\mathrm{dt}_{1}}=0$

$\Rightarrow \quad C\left(1+\frac{t_{1}}{T}+\frac{\alpha t_{1}^{3}}{1}+\frac{\alpha t_{1}^{4}}{T}-e^{\frac{t_{1}}{T}}\right)+h\left(t_{1}+\frac{t_{1}^{2}}{T}+\frac{3 \alpha t_{1}^{5}}{4 T}+\frac{3 \alpha t_{1}^{4}}{4}\right)$ $+\gamma\left(\frac{\mathrm{t}_{1}^{2}}{2}+\frac{\mathrm{t}_{1}^{3}}{2 \mathrm{~T}}+\frac{3 \alpha \mathrm{t}_{1}^{5}}{10}+\frac{3 \alpha \mathrm{t}_{1}^{6}}{10 \mathrm{~T}}\right)+\mathrm{c}_{2}\left(\mathrm{Te}^{\frac{\mathrm{t}_{1}}{T}}-\mathrm{t}_{1} \mathrm{e}^{\frac{\mathrm{t}_{1}}{\mathrm{~T}}}\right)=0$

For minimum value of $K\left(t_{1}\right) \frac{\partial K}{\partial t_{1}}=0$, which gives

$C\left[1+t_{1}+\alpha t_{1}^{\beta}+\alpha t_{1}^{\beta+1}-e^{t_{1}}\right]+h\left[t_{1}+t_{1}^{2}+\frac{\alpha \beta t_{1}^{\beta+1}}{\beta+1}+\frac{\alpha \beta t_{1}^{\beta+1}}{\beta+1}\right]$

$+\gamma\left[\frac{\mathbf{t}_{1}^{2}}{2}+\frac{\mathbf{t}_{1}^{3}}{2}+\frac{\alpha \beta \mathbf{t}_{1}^{\beta+2}}{2(\beta+2)}+\frac{\alpha \beta \mathbf{t}_{1}^{\beta+3}}{2(\beta+2)}\right]$

$+\mathrm{C}_{2}\left(\mathrm{Te}^{\mathrm{t}_{1}}-\mathrm{t}_{1} \mathrm{e}^{\mathrm{t}_{1}}\right)=\mathrm{O}$

Case II. When $\mathrm{T}=1$, the total average cost is

$\mathrm{K}\left(\mathrm{t}_{1}\right)=\frac{\mathrm{Cd}}{\mathrm{e}-1}\left[\mathrm{t}_{1}+\frac{\mathrm{t}_{1}^{2}}{2}+\frac{\alpha \mathrm{t}_{1}^{\beta+1}}{\beta+1}+\frac{\alpha \mathrm{t}_{1}^{\beta+2}}{\beta+2}-\mathrm{e}^{\mathrm{t}_{1}}+1\right]$

$+\frac{h d}{\mathrm{e}-1}\left[\frac{\mathrm{t}_{1}^{2}}{2}+\frac{\mathrm{t}_{1}^{3}}{3}+\frac{\alpha \beta \mathrm{t}_{1}^{\beta+3}}{(\beta+1)(\beta+3)}+\frac{\alpha \beta \mathrm{t}_{1}^{\beta+2}}{(\beta+1)(\beta+2)}\right]$

$+\frac{\gamma d}{e-1}\left[\frac{t_{1}^{3}}{6}+\frac{t_{1}^{4}}{8}+\frac{\alpha \beta \mathrm{t}_{1}^{\beta+3}}{2(\beta+2)(\beta+3)}+\frac{\alpha \beta \mathrm{t}_{1}^{\beta+4}}{2(\beta+2)(\beta+4)}\right]$

$+\frac{\mathrm{C}_{2} \mathrm{~d}}{\mathrm{e}-1}\left[2 \mathrm{e}^{\mathrm{t}_{1}}-\mathrm{t}_{1} \mathrm{e}^{\mathrm{t}_{1}}-\mathrm{e}\right]$

Sub-case 1. When $\beta=1$, then deterioration rate become constant.

$$
\begin{aligned}
& K\left(t_{1}\right)=\frac{c d}{e-1}\left[t_{1}+\frac{t_{1}^{2}}{2}+\frac{\alpha t_{1}^{2}}{2}+\frac{\alpha t_{1}^{3}}{3}-e^{t_{1}}+1\right]+ \\
& \frac{h d}{e-1}\left[\frac{t_{1}^{2}}{2}+\frac{t_{1}^{3}}{3}+\frac{\alpha t_{1}^{4}}{8}+\frac{\alpha t_{1}^{3}}{6}\right]+\frac{\gamma d}{e-1}\left[\frac{t_{1}^{3}}{6}+\frac{t_{1}^{4}}{8}+\frac{\alpha t_{1}^{4}}{24}+\frac{\alpha t_{1}^{5}}{30}\right]+ \\
& \frac{C_{2} d}{e-1}\left[2 e^{t_{1}}-t_{1} e^{t_{1}}-e\right] \quad \ldots . .(1.27) \\
& \frac{d K\left(t_{1}\right)}{d t_{1}}=\frac{c d}{e-1}[ \\
& +\frac{h d}{e-1}\left[t_{1}+\alpha t_{1}+\alpha t_{1}^{2}-e^{t_{1}}\right] \\
& +\frac{\gamma d}{e-1}\left[\frac{\alpha t_{1}^{3}}{2}+\frac{\alpha t_{1}^{2}}{2}\right] \\
& +\frac{C_{2} d}{e-1}\left[e^{t_{1}}-t_{1} e^{t_{1}}\right]
\end{aligned}
$$

For minimum total average cost $\frac{\mathbf{d K}\left(\mathbf{t}_{1}\right)}{\mathbf{d t}_{1}}=\mathbf{O}$

$\Rightarrow C\left(1+t_{1}+\alpha t_{1}+\alpha t_{1}^{2}-e^{t_{1}}\right)+h\left(t_{1}+t_{1}^{2}+\frac{\alpha t_{1}^{3}}{2}+\right.$ $\alpha t 122+\gamma t 122+t 132+\alpha t 136+\alpha t 146+$ C2et $1-t 1 e t 1=0$ (1.28)

$\underline{\text { Sub - Case } 2 .}$.

When $\beta=2$, deteriration rate become variable linear function of time then total average cost is

$K\left(t_{1}\right)=\frac{c d}{e-1}\left[t_{1}+\frac{t_{1}^{2}}{2}+\frac{\alpha t_{1}^{3}}{3}+\frac{\alpha t_{1}^{4}}{4}-e^{t_{1}}+1\right]+$

$\frac{h d}{e-1}\left[\frac{t_{1}^{2}}{2}+\frac{t_{1}^{3}}{3}+\frac{2 \alpha t_{1}^{5}}{15}+\frac{\alpha t_{1}^{4}}{6}\right]+\frac{\gamma d}{e-1}\left[\frac{t_{1}^{3}}{6}+\frac{t_{1}^{4}}{8}+\frac{\alpha t_{1}^{5}}{20}+\frac{\alpha t_{1}^{6}}{24}\right]+$

$\frac{C_{2} d}{e-1}\left[2 e^{t_{1}}-t_{1} e^{t_{1}}-e\right]$

For minimum value of $K\left(t_{1}\right)$, $\frac{d K\left(t_{1}\right)}{d t_{1}}=0$

$\Rightarrow C\left(1+t_{1}+\alpha t_{1}^{2}+\alpha t_{1}^{3}-\right.$

$\left.e^{t_{1}}\right)+h\left(t_{1}+t_{1}^{2}+\frac{2 \alpha t_{1}^{4}}{3}+\right.$

Published By:

Blue Eyes Intelligence Engineering

\& Sciences Publication

(C) Copyright: All rights reserved. 
$\left.\frac{\alpha t_{1}^{3}}{3}\right)+\gamma\left(\frac{t_{1}^{2}}{2}+\frac{t_{1}^{3}}{2}+\frac{\alpha t_{1}^{4}}{4}+\frac{\alpha t_{1}^{5}}{4}\right)+C_{2}\left(e^{t_{1}}-t_{1} e^{t_{1}}\right)=0$

........(1.30)

Sub-case 3. When $\beta=3$, then deterioration rate become quadratic function of time then toatl average cost is

$K\left(t_{1}\right)=\frac{c d}{e-1}\left[t_{1}+\frac{t_{1}^{2}}{2}+\frac{\alpha t_{1}^{4}}{4}+\frac{\alpha t_{1}^{5}}{5}-e^{t_{1}}+1\right]+$

$\frac{h d}{e-1}\left[\frac{t_{1}^{2}}{2}+\frac{t_{1}^{3}}{3}+\frac{\alpha t_{1}^{6}}{8}+\frac{3 \alpha t_{1}^{5}}{20}\right]+\frac{\gamma d}{e-1}\left[\frac{t_{1}^{3}}{6}+\frac{t_{1}^{4}}{8}+\frac{\alpha t_{1}^{6}}{20}+\frac{3 \alpha t_{1}^{7}}{70}\right]+$

$\frac{C_{2} d}{e-1}\left[2 e^{t_{1}}-t_{1} e^{t_{1}}-e\right]$

......(1.31)

$\frac{d K\left(t_{1}\right)}{d t_{1}}=\frac{C d}{e-1}\left(1+t_{1}+\alpha t_{1}^{3}+\alpha t_{1}^{4}-e^{t_{1}}\right)+\frac{h d}{e-1}\left(t_{1}+t_{1}^{2}+\right.$

$3 \alpha t 154+3 \alpha t 144+\gamma d e-1 t 122+t 132+3 \alpha t 1510+3 \alpha t 161$

$0+C 2 d e-1 e t 1-t 1 e t 1=0$

........(1.32)

For minimum value of $K\left(t_{1}\right) \frac{\partial K}{\partial t_{1}}=0$, which gives

$\Rightarrow C\left(1+t_{1}+\alpha t_{1}^{3}+\alpha t_{1}^{4}-e^{t_{1}}\right)+h\left(t_{1}+t_{1}^{2}+\frac{3 \alpha t_{1}^{5}}{4}+\right.$

$3 \alpha t 144+\gamma t 122+t 132+3 \alpha t 1510+3 \alpha t 1610+C 2 e t 1-t 1 e t$

$1=0$

Case III. If $\gamma=0$, then holding cost become constant. The toatl average cost is

$\mathrm{K}\left(\mathrm{t}_{1}\right)=\frac{\mathrm{cd}}{\mathrm{T}^{2}(\mathrm{e}-1)}\left[\mathrm{t}_{1}+\frac{\mathrm{t}_{1}^{2}}{2 \mathrm{~T}}+\frac{\alpha \mathrm{t}_{1}^{\beta+1}}{\beta+1}+\frac{\alpha \mathrm{t}_{1}^{\beta+2}}{\mathrm{~T}(\beta+2)}-\mathrm{Te}^{\frac{\mathrm{t}_{1}}{\mathrm{~T}}}+\mathrm{T}\right]$
$+\frac{\mathrm{hd}}{\mathrm{T}^{2}(\mathrm{e}-1)}\left[\frac{\mathrm{t}_{1}^{2}}{2}+\frac{\mathrm{t}_{1}^{3}}{3 \mathrm{~T}}+\frac{\alpha \beta \mathrm{t}_{1}^{\beta+3}}{\mathrm{~T}(\beta+1)(\beta+3)}+\frac{\alpha \beta \mathrm{t}_{1}^{\beta+2}}{(\beta+1)(\beta+2)}\right]$
$+\frac{\mathrm{c}_{2} \mathrm{~d}}{\mathrm{~T}(\mathrm{e}-1)}\left(2 \mathrm{Te} \mathrm{e}^{\frac{\mathrm{t}_{1}}{\mathrm{~T}}}-\mathrm{t}_{1} \mathrm{e}^{\frac{\mathrm{t}_{1}}{\mathrm{~T}}}-\mathrm{eT}\right)$

For minimum total averages cost, $\frac{\mathrm{dK}\left(\mathrm{t}_{1}\right)}{\mathrm{dt}_{1}}=\mathbf{O}$

$\Rightarrow \quad C\left(1+\frac{t_{1}}{T}+\alpha t_{1}^{\beta}+\frac{\alpha t_{1}^{\beta+1}}{T}-e^{\frac{t_{1}}{T}}\right)+h\left(t_{1}+\frac{t_{1}^{2}}{T}+\frac{\alpha \beta t_{1}^{\beta+2}}{T(\beta+1)}+\frac{\alpha \beta t_{1}^{\beta+1}}{(\beta+1)}\right)$

$+c_{2}\left(T e^{\frac{t_{1}}{T}}-t_{1} e^{\frac{t_{1}}{T}}\right)=0$

Sub-case 1. When $\beta=1$, deteriration rate become constant. $K\left(t_{1}\right)=\frac{C d}{T^{2}(e-1)}\left[t_{1}+\frac{t_{1}^{2}}{2 T}+\frac{\alpha t_{1}^{2}}{2}+\frac{\alpha t_{1}^{3}}{3 T}-T^{\frac{t_{1}}{T}}+T\right]$ $+\frac{\text { hd }}{T^{2}(e-1)}\left[\frac{t_{1}^{2}}{2}+\frac{t_{1}^{3}}{3 T}+\frac{\alpha t_{1}^{4}}{\delta T}+\frac{\alpha t_{1}^{3}}{6}\right]$ $+\frac{\mathrm{C}_{2} \mathrm{~d}}{\mathrm{~T}(\mathrm{e}-1)}\left[2 \mathrm{~T} \mathrm{e}^{\frac{\mathrm{t}_{1}}{\mathrm{~T}}}-\mathrm{t}_{1} \mathrm{e}^{\frac{\mathrm{t}_{1}}{\mathrm{~T}}}-\mathrm{eT}\right]$

For minimum value of $K\left(t_{1}\right)$, $\frac{d K\left(t_{1}\right)}{{d t_{1}}_{1}}=0$

$\Rightarrow \quad C\left(1+\frac{t_{1}}{T}+\alpha t_{1}+\frac{\alpha t_{1}^{2}}{T}-e^{\frac{t_{1}}{T}}\right)+h\left(t_{1}+\frac{t_{1}^{2}}{T}+\frac{\alpha t_{1}^{3}}{2 T}+\frac{\alpha t_{1}^{2}}{2}\right)$

$+C_{2}\left(T e^{\frac{t_{1}}{T}}-t_{1} e^{\frac{t_{1}}{T}}\right)=0$

Sub- case 2 . When $\beta=2$, deteriration rate become variable linear function of time.

$$
\begin{aligned}
& \mathrm{K}\left(\mathrm{t}_{1}\right)=\frac{\mathrm{Cd}}{\mathrm{T}^{2}(\mathrm{e}-1)}\left[\mathrm{t}_{1}+\frac{\mathrm{t}_{1}^{2}}{2 \mathrm{~T}}+\frac{\alpha \mathrm{t}_{1}^{3}}{3}+\frac{\alpha \mathrm{t}_{1}^{4}}{4 \mathrm{~T}}-\mathrm{Te}^{\frac{\mathrm{t}_{1}}{\mathrm{~T}}}+\mathrm{T}\right] \\
& +\frac{\mathrm{hd}}{\mathrm{T}^{2}(\mathrm{e}-1)}\left[\frac{\mathrm{t}_{1}^{2}}{2}+\frac{\mathrm{t}_{1}^{3}}{3 \mathrm{~T}}+\frac{2 \alpha \mathrm{t}_{1}^{5}}{15 \mathrm{~T}}+\frac{\alpha \mathrm{t}_{1}^{4}}{6}\right]
\end{aligned}
$$

$$
+\frac{C_{2} d}{T(e-1)}\left[2 T^{2} e^{\frac{t_{1}}{T}}-t_{1} T e^{\frac{t_{1}}{T}}-e T^{2}\right]
$$

For minimum value of $\mathrm{K}\left(\mathrm{t}_{1}\right)$, $\frac{\mathrm{dK}\left(\mathrm{t}_{1}\right)}{\mathrm{dt}_{1}}=\mathbf{O}$

$$
\begin{aligned}
& \Rightarrow \quad C\left(1+\frac{t_{1}}{T}+\alpha t_{1}^{2}+\frac{\alpha t_{1}^{3}}{T}-e^{\frac{t_{1}}{T}}\right)+h\left(t_{1}+\frac{t_{1}^{2}}{T}+\frac{2 \alpha t_{1}^{4}}{3 T}+\frac{2 \alpha t_{1}^{3}}{3}\right) \\
& +C_{2}\left(T e^{\frac{t_{1}}{T}}-t_{1} e^{\frac{t_{1}}{T}}\right)=0
\end{aligned}
$$

Sub-case 3 . When $\beta=2$, deteriration rate become quadratic function of time then total average cost is

$$
\begin{aligned}
& \mathrm{K}\left(\mathrm{t}_{1}\right)=\frac{\mathrm{Cd}}{\mathrm{T}^{2}(\mathrm{e}-1)}\left[\mathrm{t}_{1}+\frac{\mathrm{t}_{1}^{2}}{2 \mathrm{~T}}+\frac{\alpha \mathrm{t}_{1}^{4}}{4}+\frac{\alpha \mathrm{t}_{1}^{5}}{5 \mathrm{~T}}-\mathrm{Te}^{\frac{\mathrm{t}_{1}}{\mathrm{~T}}}+\mathrm{T}\right] \\
& +\frac{\mathrm{hd}}{\mathrm{T}^{2}(\mathrm{e}-1)}\left[\frac{\mathrm{t}_{1}^{2}}{2}+\frac{\mathrm{t}_{1}^{3}}{3 \mathrm{~T}}+\frac{\alpha \mathrm{t}_{1}^{6}}{8 \mathrm{~T}}+\frac{3 \alpha \mathrm{t}_{1}^{5}}{2 \mathrm{O}}\right] \\
& +\frac{\mathrm{C}_{2} \mathrm{~d}}{\mathrm{~T}(\mathrm{e}-1)}\left[2 \mathrm{~T} \mathrm{e}^{\frac{\mathrm{t}_{1}}{\mathrm{~T}}}-\mathrm{t}_{1} \mathrm{Te} \mathrm{e}^{\frac{\mathrm{t}_{1}}{\mathrm{~T}}}-\mathrm{e} \mathrm{T}\right] \ldots \ldots(1.40)
\end{aligned}
$$

For minimum total average cost, $\frac{\mathrm{dK}\left(\mathrm{t}_{1}\right)}{\mathrm{dt}_{1}}=\mathbf{O}$,

$$
\begin{aligned}
& \Rightarrow \quad C\left(1+\frac{t_{1}}{T}+\alpha t_{1}^{3}+\frac{\alpha t_{1}^{4}}{T}-e^{\frac{t_{1}}{T}}\right)+h\left(t_{1}+\frac{t_{1}^{2}}{T}+\frac{3 \alpha t_{1}^{5}}{4 T}+\frac{3 \alpha t_{1}^{4}}{4}\right) \\
& +C_{2}\left(T e^{\frac{t_{1}}{T}}-t_{1} e^{\frac{t_{1}}{T}}\right)=0
\end{aligned}
$$

\section{CONCLUSION}

In the paper, we have attempted to evolve a two-warehouse inventory structure too an exceptionally reasonable practical disintegrating rate. The impact of debilitating of physical items in stock is extraordinarily reasonable component's stock control. Now in the model decay rate at anything is relied upon to seek after two parameter Weibull scattering limit of time. This debilitating rate is appropriate for things with and without life-period. The two warehouse stock issue is a beguiling yet feasible purpose of decision science. The two-warehouse model can be associated with various sensible conditions, in light of introduction of open market technique; the business contention ends up being very high to have most prominent possible market.

\section{REFERENCES}

1. Aggarwal, S.P. and Jaggi, C.K., 1995. Ordering policies of deteriorating items under permissible delay in payments. J.O.R.S. , 46.

2. Chakrabarty, T., Giri, B.C., Chaudhuri, K.S., 1998. An EOQ model for items with weibull distribution deterioration, shortages and trended demand: an extension of Philip's model. C.O.R. 25

3. Covert, R.P. and Philip, G.P., 1973. An EOQ model for items with Weibull distribution deterioration. AIIE Trans., 5.

4. Ghare, P.M. and Schrader, G.P., 1963. A model for exponentially decaying inventory. J.I.E. 4.

5. Goyal, S.K., 2015. Economic order quantity under conditions of permissible delay in payments. J.O.R. 36

6. Bansal K.K., Ahlawat N. [2012] Integrated Inventory Models for Decaying Items with Exponential Demand under Inflation. International Journal of Soft Computing and Engineering (IJSCE) 2[3] 578-587.

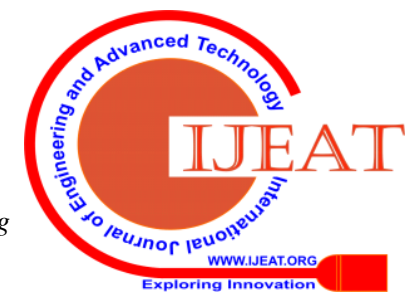


7. Bansal K.K. [2016] A Supply Chain Model With Shortages Under Inflationary Environment. Uncertain Supply Chain Management 4[4] 331-340

8. Kalpana, Dr.Kapil Kumar Bansal (2017) : Inventory Model For Decaying Items With Partial Backlogging And Inflation Under Trade Credits, International Journal of Education and Science Research ReviewVol.2 (6)

9. Bansal K.K ,Pravinder Kumar (2015) : Developing and Measuring Supply Chain Management \& Logistics Concepts In India International Journal Of Advanced Research In Engineering Technology \& Sciences Vol.2(10)

10. Sharma M.K.,Bansal K.K.[2017] Inventory Model for NonInstantaneous Decaying Items with Learning Effect under Partial Backlogging and Inflation. Global Journal of Pure and Applied Mathematics.vol.13 [6] pp. 1999-2008

11. Philip, G.C., 1974. A generalize EOQ model for items with Weibull distribution deterioration. AIIE Trans., 6.

12. Pakkala, T.P.M., Achary, K.K., 1992a. Discrete time inventory model for deteriorating items with two warehouses. Opsearch 29.

13. S.R. Singh and T.J. Singh, 2008. Perishable inventory model with quadratic demand, partial backlogging and permissible delay in payments, I.R.P.A. Mathematics, 1.

14. Sarma K.V.S., 1987. A deterministic inventory model for deteriorating items with two storage facilities. E.J.O.R. 29.

15. Singh, S.R. and Singh, S., 2009. "A profit maximizing inventory model having exponentially increasing demand under trade credit", J.A.B.M, $1,2$.

16. Singh, S.R. and Singh, S., 2008. "Production model for items under the effect of inflation and permissible delay in payments", I.R.P. Mathematics, 4, 1.

17. Yang, H.L., 2006. Two-warehouse partial backlogging inventory models for deteriorating items under inflation. I.J.P.E., 103.

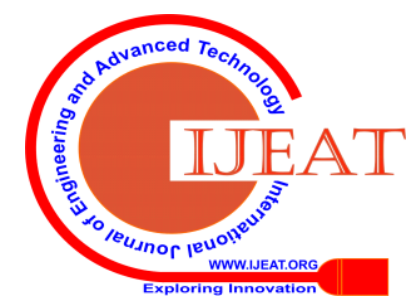

